\title{
Reflective Power Limiter for X-band with HTSC switching element
}

\author{
B. A. Belyaev, I.V. Govorun, A. A. Leksikov, A.M. Serzhantov, and An. A. Leksikov ${ }^{1}$
}

\begin{abstract}
This paper presents a new type of power limiter (PL), using superconducting-to-normal state phase transition in HTSC. The presented device differs from the existing ones mainly in the reflective principle of its operation. In fact the PL is a three-resonator microstrip filter, whose resonators are configured and arranged in such a manner that coupling coefficient between the outer resonators equals zero, and the inner one has a gap in the middle section shunted by a HTS film element. In small signal mode, the HTS element is in low-loss state, and the device is essentially a three-pole filter with low transmission loss. When the level of the signal exceeds the threshold, HTS element transits to the high-loss state, and $Q$-factor of the inner resonator falls, breaking the coupling in the structure. This leads to the corresponding increase in PL's transmission loss due to reflection. The more the level of incoming power, the more does the $Q$-factor fall. The PL operating frequency is $8 \mathrm{GHz}$ with $8.5 \%$ fractional bandwidth. It remains operable up to $40 \mathrm{~W}$ of input power, providing $38 \mathrm{~dB}$ limitation.
\end{abstract}

Index Terms - power limiter, microwave, transmission zero, microstrip structure

\section{INTRODUCTION}

A mong the challenges that confront the developers in designing the radar system, the problem of protecting the system's input circuits from both external damaging microwave pulse and the effect of side lobes of its own transmitter antenna is well known. Many engineers proposed various ideas for construction of microwave power limiters that dampen the impact of damaging pulses. Among these, the most widely used ones are the semiconductor limiters, based on diodes or varactors [1]-[6], but they also sometimes fail to satisfy the modern requirements, with respect to robustness and response speed. The recent development of microwave power limiters (PLs), based on RF-MEMS technology [7], makes it possible to increase the response speed, but they have, at the moment, only around $10 \mathrm{~dB}$ of signal suppression. Quite good characteristics (short switching time, high level of power limitation, and high robustness) are achieved with vacuum electron devices by employing transverse electron bunching under cyclotron resonance conditions [8],[9]. However, these devices are rather difficult to manufacture and adjust. Besides, they are weighty and voluminous, particularly because their operation requires constant, high and homogeneous magnetic field.

B. A. Belyaev, A. A. Leksikov, A.M. Serzhantov are with Kirensky Institute of Physics SB RAS, Krasnoyarsk, 660036, Russia and Siberian Federal University, Krasnoyarsk, 660074, Russia

I. V. Govorun and An. A. Leksikov are with Kirensky Institute of Physics SB RAS, Krasnoyarsk, 660036, Russia (e-mail: a.a.leksikov@gmail.com)
The development of high-temperature superconductors (HTSCs), which are characterized by an extremely short time $\left(<10^{-10} \mathrm{~s}\right)[10]$ to switch from a lowloss superconducting state to a high-loss normal state, stimulated further research on their possible use in power limiter systems. Usually, the HTSC-based device comprises a segment of strip, microstrip, or coplanar transmission lines made from HTSC materials that are matched with the tract [11]-[14]. The operating principle of these devices is obvious: when transmitted signal generates in the line a current density that exceeds a critical level, the material switches from the superconducting to normal (high-loss) state, which leads to strong attenuation of output power. However, sufficient part of the power, which is not transmitted to the output, is absorbed by the transmission line as Joule heat. For this reason, the robustness of these devices is rather low, and it is determined primarily by the efficiency of heat removal from the system.

For further development of the HTSC-based approach, [17], [18] authors tried to combine PL with another important component of radar system - bandpass filter. It is known that the size of a radar system, especially airborne radar system, is determined largely by the size of the bandpass filters. So, this solution promises several advantages. The PLs having bandpass filter structure will reduce the size of the system, as the device simultaneously works as a filter, and needs fewer amplifiers, because a bandpass filter with HTSC resonators has a very small transmission loss. Besides, the robustness of the device improves as the operating principle of the device changes from mainly absorptive to mainly reflective. In other words, when a high-power pulse switches an input resonator of the bandpass filter into the high-loss state, the unloaded quality factor of the resonator dramatically decreases, while transmission loss significantly increases, largely due to reflection. One can see it clearly in graphs in ref [18].

However, from the same graphs, it can be seen that such degradation of the pass-band decreases the reflection loss to a level of around $-3 \mathrm{~dB}$. This means that up to half

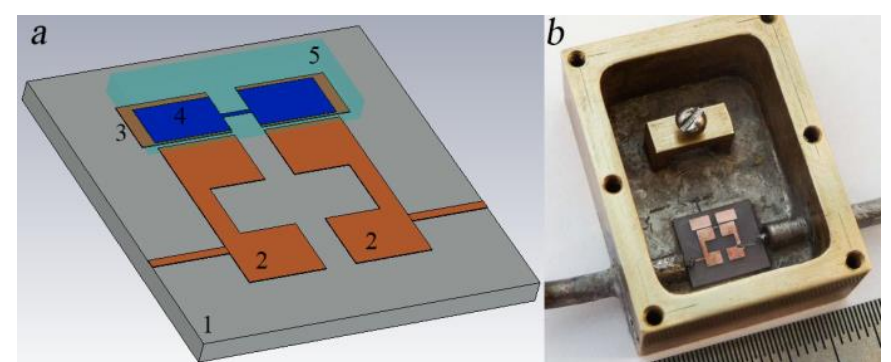

Fig. 1. Design of microstrip reflective power limiter (a):1 - Dielectric substrate; 2 - Copper external resonator; 3 - Copper parts of internal resonator; 4 - HTSC element; $5-\mathrm{NdGaO}_{3}$ substrate. (b) Photograph of the device's experimental realization for $\mathrm{X}$-band 


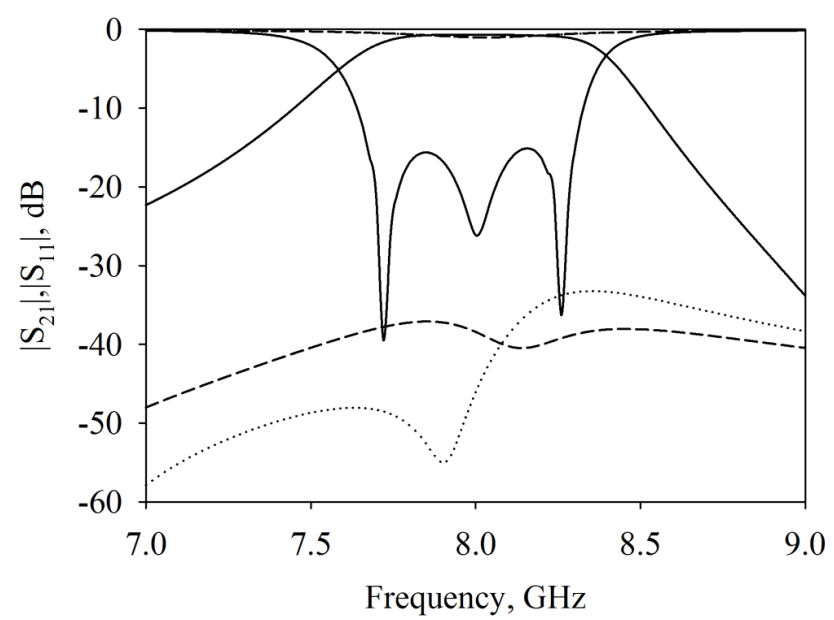

Fig. 2. Frequency response of a power limiter in the open state (solid line), and closed state (dashed line), in the absence of internal resonator and HTSC element (dotted line)

of the power is absorbed in the input resonator; thus, the improvement in robustness of the device remains still inadequate.

Also, diode and HTSC-based PLs are nonlinear devices, and at the level of input signal around $+63 \mathrm{dBm}$ [11], the first and the third harmonics already have the same levels. This suggests that one needs to take into account the signal generated in the power limiter.

Previously, we [19] proposed a PL, based on the 3pole microstrip bandpass filter (BPF), where only the central part of the internal resonator was made from HTSC thin film and the remaining parts from copper. When the superconducting part of the resonator is in the low-loss state, all the resonators form a pass-band, while in the high-loss state there is no coupling between the external resonators and the internal one, and hence most part of the incoming signal power reflects back. Workability of the proposed idea was proved by the PL with a central frequency of $2 \mathrm{GHz}$ that has operating bandwidth $17.5 \%$ and suppression level of more than 20 $\mathrm{dB}$ in the closed state.

In the present paper, we describe a PL with central frequency $8 \mathrm{GHz}$ and then present the results of its investigation, with special reference to operation at higher power, pulse mode, and the nonlinear properties of the device.

The remainder of the paper is organized as follows. Operating principle of the power limiter is described in Section II and the construction of the power limiter for Xband in Section III. The transmission characteristics of the fabricated PL were measured in continuous signal mode at higher power (Section IV) and in pulse signal mode of different time durations (Section V). The peculiarities of nonlinear PL's behavior are discussed in Section VI.

\section{Principle of Power Limiter Operation}

In microwave theory, the frequency-dependent coupling coefficients [20], inductive and capacitive ones, describe magnetic and electric interactions between two microwave resonators. According to this theory, the total coupling coefficient $k(f)$ is:

$$
k(f)=\frac{k_{L}(f)-k_{C}(f)}{1-k_{L}(f) k_{C}(f)},
$$

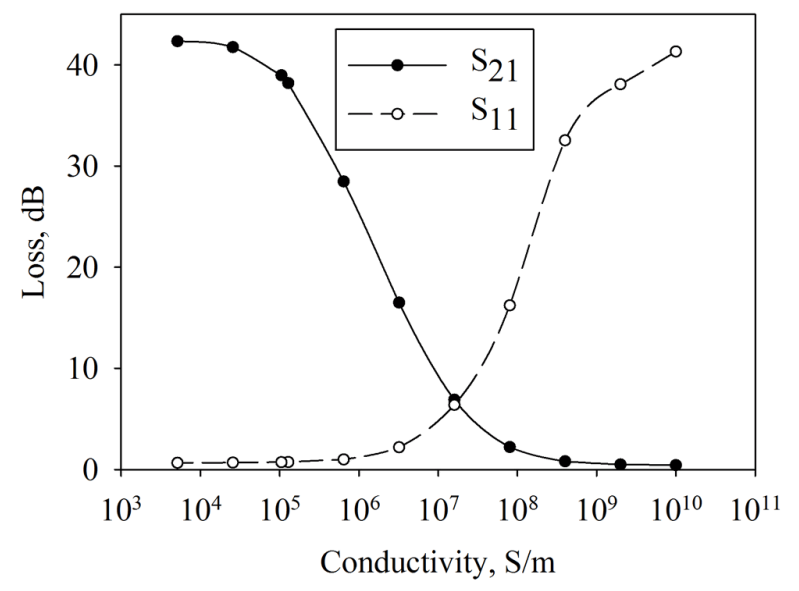

Fig. 3. Transmission and reflection loss dependencies versus conductivity of HTSC element in the power limiter

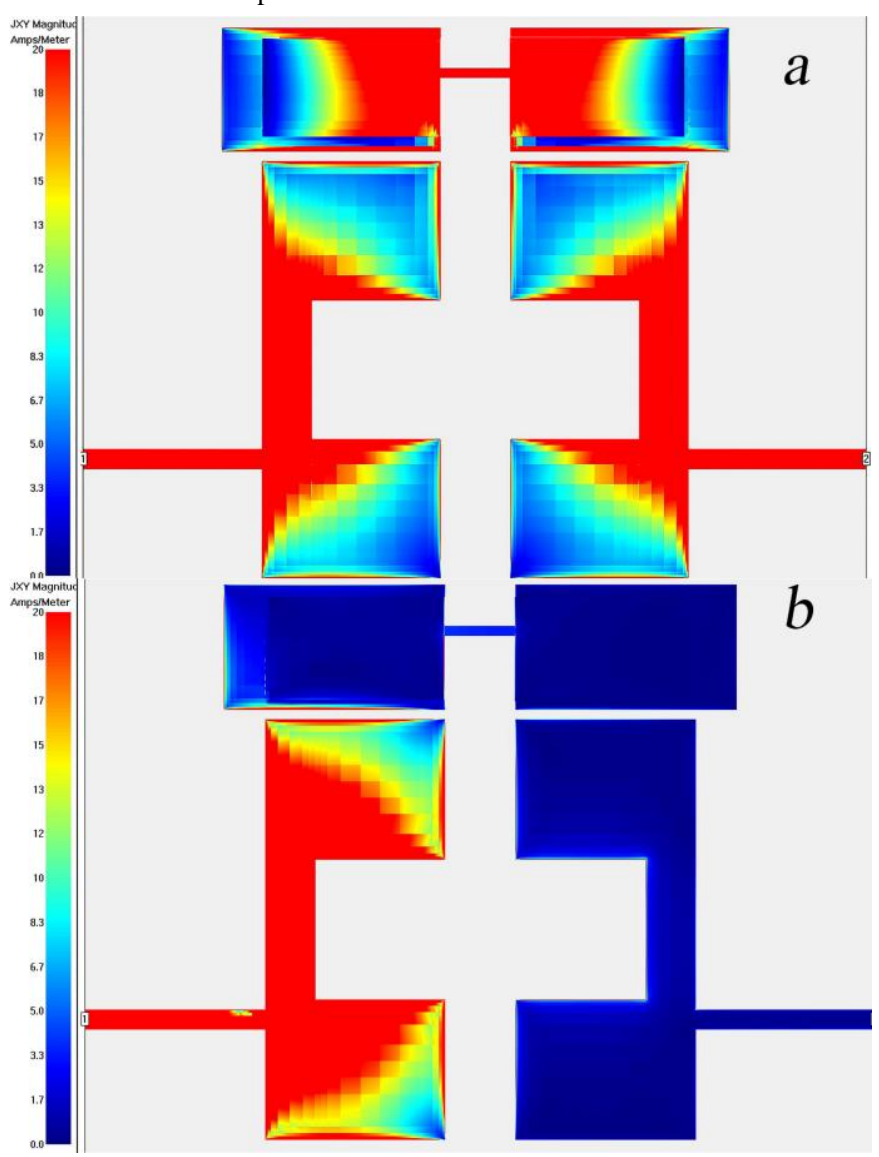

Fig. 4. Simulated distribution of microwave currents' density in PL in open mode $(a)$ and limitation mode $(b)$.

where $k_{L}(f)$ and $k_{C}(f)$ are inductive and capacitive coupling coefficients, reflecting interactions through magnetic and electric fields, respectively. The frequency-dependence of these coefficients originates from individual distribution of voltage and current (as well as magnetic and electric fields) along the resonator's strip at every frequency. Earlier [19], it was shown that, by choosing a resonator shape and a gap between resonators, one can achieve equality between inductive $k_{L}$ and capacitive $k_{C}$ coupling coefficients at some frequency, which means zeroing of total coupling coefficient. When this occurs, a damping pole (a sharp minimum of transmission) results at this frequency. From physical point of view, this means that, at this frequency, magnetic (inductive) interaction requires that MW currents in the resonators flow in the same direction, whereas electric (capacitive) interaction requires them to flow in opposite. This leads to total MW 


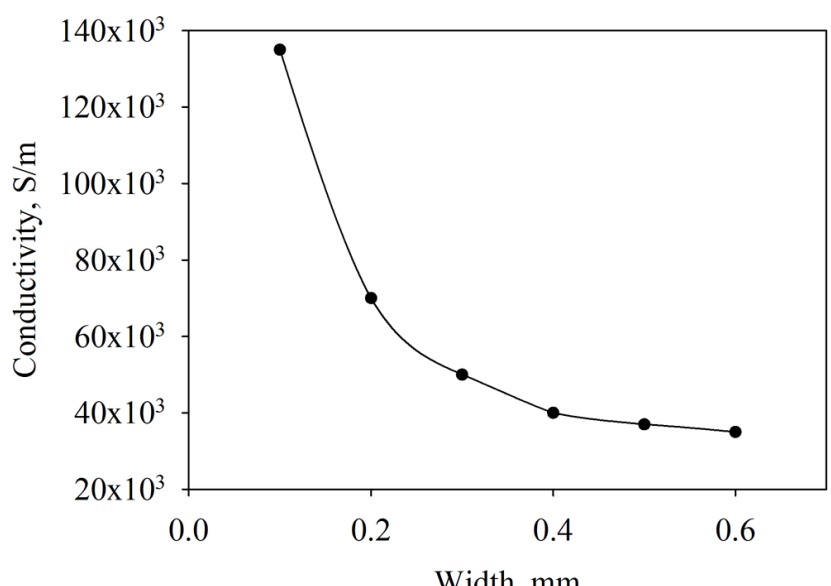

Fig. 5. Threshold value of HTSC element's conductivity, corresponding to transmission zero appearance on the frequency response of the device versus the bridge's width

reflection from the input port of the structure at that frequency. Moreover, by proper choice of the structure's parameters, one can shift the transmission zero (damping pole) to a resonant frequency of the structure. It means that, instead of a pass-band in the frequency response of the structure, one will observe strong reflection at the resonant frequency from the input port.

The BPF-based power limiter (Fig. 1a) is essentially a 3-pole bandpass filter on a substrate, whose bottom surface is completely metal-coated (grounded base). The external resonators are identical in shape (C-shaped in this case) and are made of a "normal" metal (copper). The third, central one, is a composite resonator made of copper, whereas its central part has a cut, shunted by an HTSC film element. A dumbbell-shaped strip conductor of this element represents the $\mathrm{YBaCuO}$ thin film deposited onto $0.5 \mathrm{~mm} \mathrm{NdGaO}_{3}$ substrate. The external resonators' shape and the gap between them were so chosen that, in the absence of the internal resonator, a transmission zero exists at the resonance frequencies of the structure (dotted line in Fig 2). In the presence of the central resonator, when the HTSC element is switched in the high-loss state $(95 \Omega / \square)$, the unloaded quality factor of the resonator is very small; so, the coupling between the internal and external resonators could not be organized to form the pass-band (dashed line in Fig. 2). In this case, the internal resonator alters the capacitive coupling between the external ones only slightly. However, when the HTSC element switches to the low-loss state, allowing high density microwave current flow through it, the unloaded quality factor of internal resonator significantly rises and the resonator gets incorporated into the interaction inside the structure, forming a pass-band of the device (solid line in Fig. 2).

In Fig. 3 the dependencies of transmission losses $\left(\mathrm{S}_{21}\right)$ and return losses $\left(\mathrm{S}_{11}\right)$ versus conductivity of HTSC element are presented. These results were obtained by simulation of the structure in the AWR Microwave Office, taking the result at the central frequency of the pass-band. One can see in Fig. 3 that, with significant rise in transmission loss, the reflection loss dramatically drops. It means that, with decrease of HTSC's element conductivity, transmission through the structure becomes worse as the coupling between the resonators decreases, while reflection from input port increases. When the

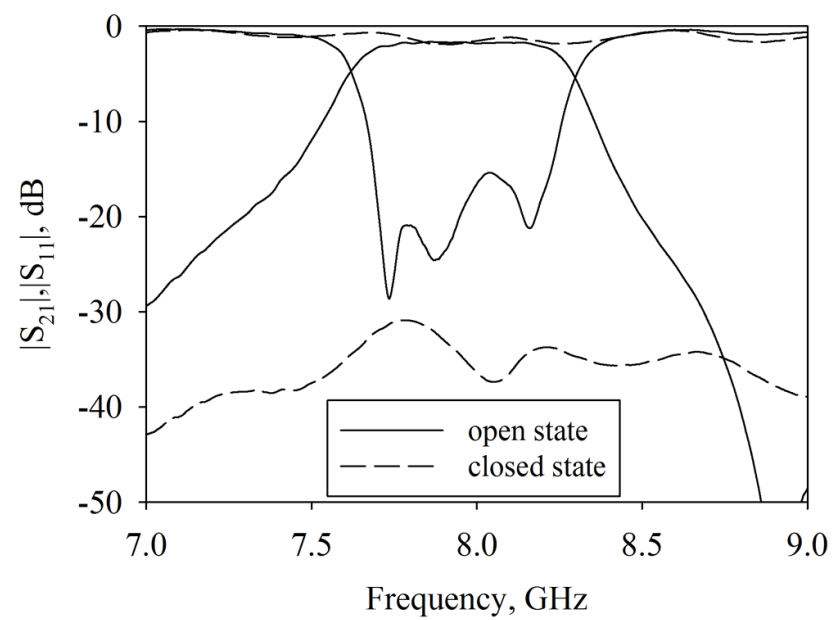

Fig. 6. Frequency response of the fabricated device, measured in both states

MW generator
R\&S SMB100A $\Rightarrow \begin{gathered}\text { Amplifier } \\ 55 \mathrm{~dB}\end{gathered} \Rightarrow \begin{aligned} & \text { Power } \\ & \text { limiter }\end{aligned} \Rightarrow \begin{gathered}\text { Spectrum analyzer } \\ \text { R\&S FSL }\end{gathered}$

Fig. 7. Scheme for transfer characteristics measurements

conductivity drops to $135 \mathrm{kSm} / \mathrm{m}$, transmission zero appears on the frequency response of the structure. Thus, at such conductivity of HTSC film, the fact that the internal resonator is not involved in the structure's operation is evidenced by the change in the slope of the dependencies presented in the figure. So it can be concluded that the proposed structure may be used in power limiters, saving its workability after expose to high power radiation, because only a small part of power has been absorbed in it, and the remainder reflected.

Surface resistivity of our HTSC thin film (in normal state) is $\rho=95 \Omega / \square$, which corresponds to conductivity $\sigma$ $=105.2 \mathrm{kSm} / \mathrm{m}$; so, the transmission loss of the structure should be, according to simulation result, around $39 \mathrm{~dB}$ and the return losses $-0.7 \mathrm{~dB}$. Therefore, $85 \%$ of the incident power is reflected from input port, and only $15 \%$ absorbed in the structure, mainly in the input resonator.

In Fig. 4. results of simulation (Sonnet) are shown which demonstrates MW currents' density distribution in the device for two cases. a) Bridge in the HTS element is in low loss (superconductive) state and PL is open: in both input and output resonators the current densities are the same. b) Bridge is in high loss state: conductivity of the bridge $\sigma=105.2 \mathrm{kSm} / \mathrm{m}$. It is seen, the current density in the output resonator drops several orders of magnitude. This means PL is in limitation mode.

Previously, [19] has shown that the leakage level in the device depends on the width of the bridge (narrow part) in the HTSC dumbbell-shaped element and it is of the order of tens $\mathrm{mW}$. It is obvious that the critical power (i.e. switching HTSC element) will also depend on bridge's width. A simulation was done when only the conductivity of central part of the HTSC element was changing, while the external part remained in the same state. The results of simulation are presented in Fig. 5. From this figure, one can see that, for the HTSC film with surface resistivity $95 \Omega / \square$ and thickness $200 \mathrm{~nm}$, the desirable width of the bridge should be less than $0.3 \mathrm{~mm}$.

A power limiter for X-band operation was designed, fabricated and tested in various modes of operation to prove the operability of the proposed idea. It should be 


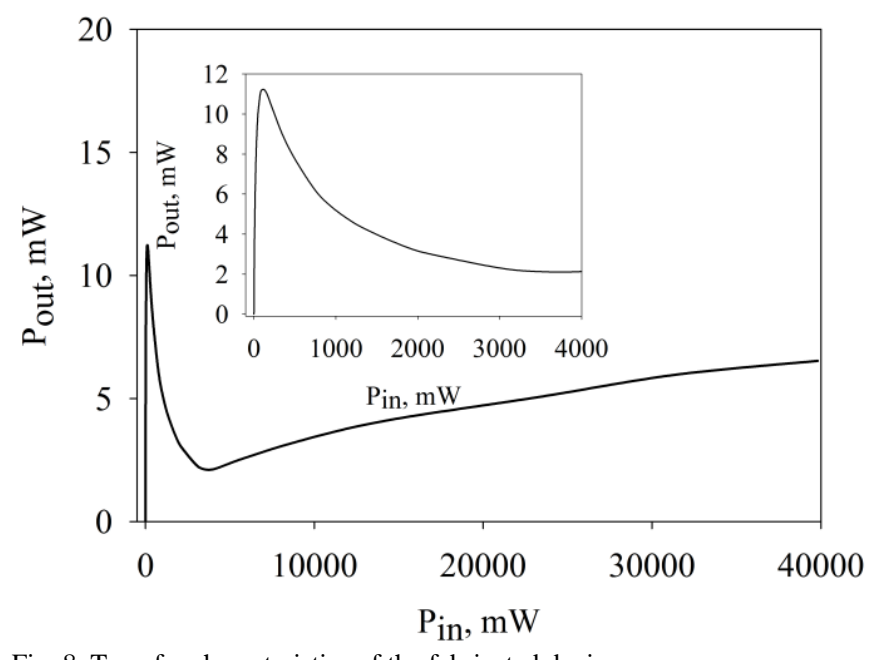

Fig. 8. Transfer characteristics of the fabricated device.

noted, in the designing process we used a technique analogous to that described in Ref. [23].

Particularly, the performance of the PL at input power higher than $10 \mathrm{~W}$, and arising distortion of the pass band in the pulse mode, were investigated. In addition, the non-linearity of the device was checked.

\section{PoWer Limiter BASED ON MiCROSTRIP RESONATORS}

The design was fulfilled as follows. Initially the sizes of the pair of input and output copper resonators were determined for a chosen operation frequency. Then a gap between the resonators was regulated to minimize transmission through the structure having a transmission zero inside the passband and two copper patches forming the third resonator were added. After that, a correction of the gap was made, because the patches bring a slight change in the interaction between the resonators and shift the position of transmission zero. Next, dumbbell-shaped HTS film element was incorporated into the structure by applying its wide parts to the patches. When HTS element is in a low-resistance state a strong capacitive connection between them and patches exists allowing formation the third resonators. Sizes of the latter are fitted to form a passband of a three-resonator filter. The next iteration consists of choosing the width of the bridge in the HTS element. This value determines limitation level in the closed state of the device. In this case conductivity of the bridge is chosen to be equal the conductivity of HTS in the normal state. When bridge's width is chosen from a required minimum of limitation the last tuning is made as this parameter influences a resonant frequency of the resonator in superconductive state.

The power limiter, based on C-shaped microstrip resonators, was designed and fabricated on an alumina substrate of thickness $0.5 \mathrm{~mm}$ and dimensions $7.9 \mathrm{~mm} \times 8.2 \mathrm{~mm}$ (see Fig. 1a). The device consists of two external resonators of an aggregate length of $4.2 \mathrm{~mm}$, and consists of three parts: two wide $(1.8 \mathrm{~mm})$ parts and one narrow part $(0.5 \mathrm{~mm})$. All these parts were of the same length. The gap between the low-impedance parts of resonators was $0.7 \mathrm{~mm}$, and that between high-impedance parts $3.3 \mathrm{~mm}$. Conductive connection of the structure to the path was obtained by means of feed lines, $0.2 \mathrm{~mm}$ in width and $1.8 \mathrm{~mm}$ in length, which were connected to the external resonators at $1.0 \mathrm{~mm}$ from the edge. The copper

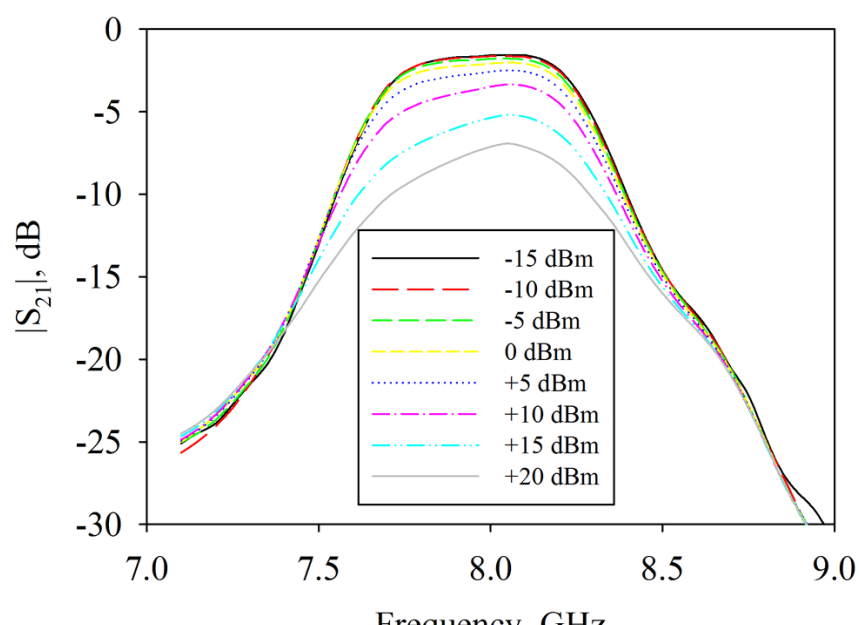

Frequency, GHz

Fig. 9. Transmission loss transformation with the signal level increasing in the case of $3 \mu$ s sweeping.

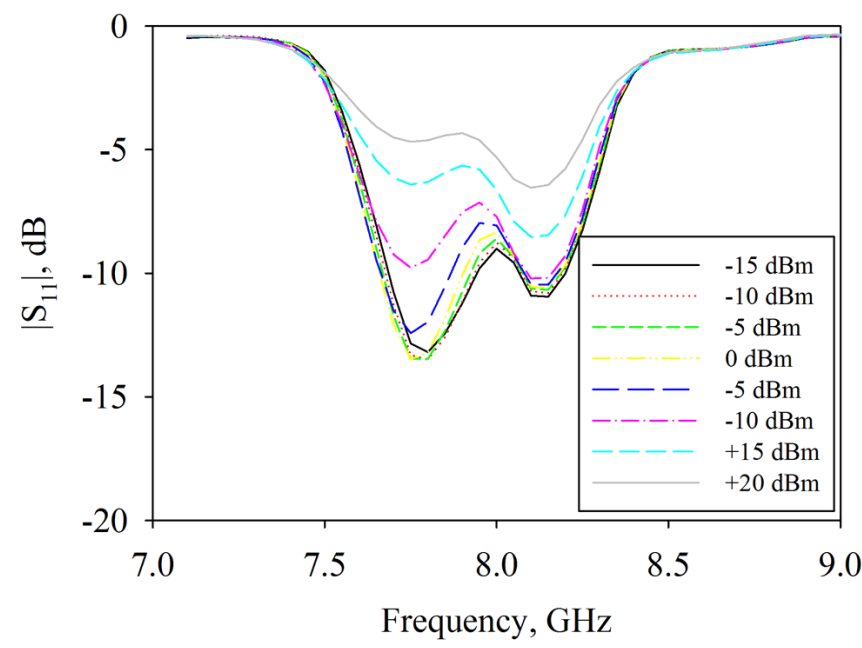

Fig. 10. Return loss transformation with the signal level increasing in the case of $3 \mu$ s sweeping.

parts of the internal resonator measured $2.1 \mathrm{~mm} \times 1.2 \mathrm{~mm}$ and were separated by $0.7 \mathrm{~mm}$ gap. The dumbbell-shaped HTSC element used in the structure had a thickness 100 $\mathrm{nm}$; its dimensions of the wide parts were $1.8 \mathrm{~mm}$ $\times 1.0 \mathrm{~mm}$ and those of the bridge $0.7 \mathrm{~mm} \times 0.1 \mathrm{~mm}$. The HTSC element had no ohmic contact with the copper parts and was attached to them only by a clamp. So, it had a "capacitive" coupling to the copper parts of the resonator, which appeared to be enough for operability of the device.

The power limiter was mounted on a brass case (Fig. 1b) measuring $31 \mathrm{~mm} \times 24 \mathrm{~mm} \times 14 \mathrm{~mm}$, using coaxial waveguides to input and output a signal.

Liquid nitrogen was used for cooling the device.

\section{TRANSMISSION AND TRANSFER Characteristics of The PoWer Limiter}

Frequency responses of the fabricated power limiter, measured with the help of vector network analyzer (VNA) R\&S ZNB, are shown in Fig. 6 for both cases: low-loss state of the HTSC element at liquid nitrogen temperature - solid line; and high-loss state of the HTSC element at room temperature - dashed line. One can see that the central frequency of the device is $7.95 \mathrm{GHz}$ with $8.5 \%$ of operational fractional bandwidth. The transmission loss in the low-loss state is $1.65 \mathrm{~dB}$, while the return loss inside 
the pass band is less than $15 \mathrm{~dB}$. The loss in the passband, as compared to that of the simulation, is rather high. This is probably because the coaxial waveguides were not taken into account during calibration. In the high-loss state, the transmission losses at the frequencies of the pass-band are higher than $30 \mathrm{~dB}$, while the return losses are only about $2 \mathrm{~dB}$. On the whole, a rather good agreement can be seen between the simulated characteristics (Fig. 2) and the measured ones (see Fig. 6).

PL's transfer characteristics were measured using the scheme shown in Fig. 7. The measurements were carried out at the temperature of liquid nitrogen, at a frequency 8 $\mathrm{GHz}$ and the results are presented graphically in Fig. 8. One can see from the graph that the PL, triggered at the input power of about $100 \mathrm{~mW}(20 \mathrm{dBm})$, gives the maximum leakage level of $11 \mathrm{~mW}(10.8 \mathrm{dBm})$. Minimal transfer of $2 \mathrm{~mW}(3 \mathrm{dBm})$ is observed at $4 \mathrm{~W}(36 \mathrm{dBm})$ of input microwave power. It seemingly corresponds to the power transfer that occurs when the whole HTSC element is switched to the high-loss state. Further increase in the transferred power corresponds to direct leakage between the input and output resonators. Nevertheless, at the input power of $40 \mathrm{~W}(46 \mathrm{dBm})$, only $6.5 \mathrm{~mW}(8 \mathrm{dBm})$ of transmitted power is observed, which corresponds to 38 dB limitation.

It should be noted that in the measurement of transfer characteristics, various HTSC films of varying thickness and surface resistivity were used in the switching element, but no hysteresis behavior was found in the device operation, as reported earlier [22].

\section{PL'S Transmission Characteristics in a PULSE MODE}

Obviously for frequency-selective devices, it is very important to save a form of pass-band at various signals' levels (in open state concerning the present case). To prove this, measurements were carried out with the help of Keysight PNA-X vector network analyzer, using an instrument that generates and measures the reflected and transmitted signals in the single sweep mode. In this mode, the PNA-X generator operates only during a chosen time, whereas in other VNAs the generators usually continue to operate even after the measurement, while only the receivers are turned off.

For pass-band observation, one needs to make frequency sweep to obtain the desired dependence. A 40point sweep was chosen to measure the behavior of passband versus input power over four different durations of sweeping: $3 \mu \mathrm{s}, 298 \mu \mathrm{s}, 5 \mathrm{~ms}$, and continuous sweep. At the shortest sweep $(3 \mu \mathrm{s})$, at each frequency point, the generator operates only $75 \mathrm{~ns}$. This information was used to estimate switch time. The frequency responses of the PL were measured at the input power ranging from -15 $\mathrm{dBm}$ to $+20 \mathrm{dBm}$, every $5 \mathrm{dBm}$, and the data is presented in Fig. 9 for transmission losses and in Fig.10 for return losses.

Several important consequences follow from the data: 1) in the pulse sweep at $+20 \mathrm{dBm}$ at the central frequency, the limitation level is $9 \mathrm{~dB}$, which agrees with the result obtained during the transfer measurement (Section IV); 2) the pass-band retains its shape until $-5 \mathrm{dBm}$; 3 ) the device switches to the limiter mode for not more than $75 \mathrm{~ns}$ $(3 \mu \mathrm{s} / 40)$. For more precise definition another technique should be used.

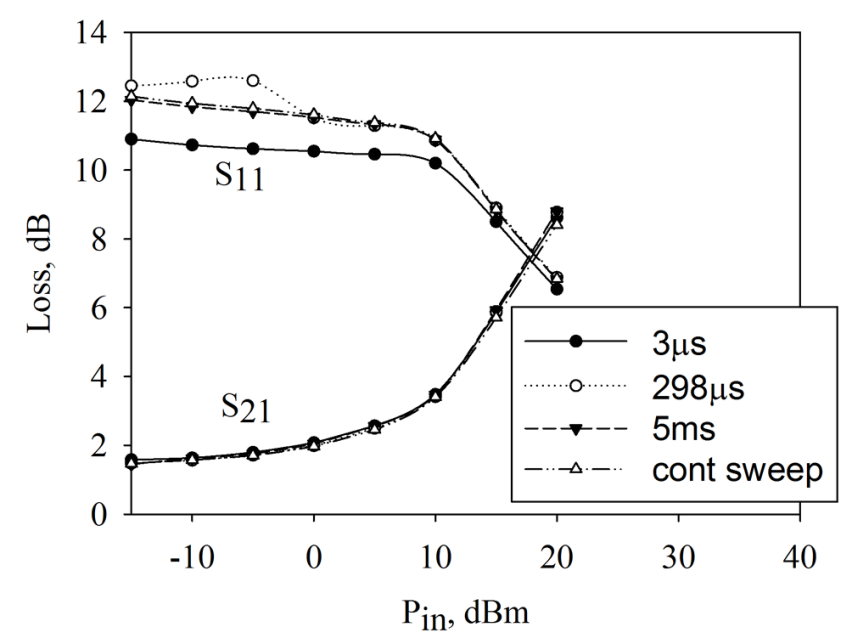

Fig. 11. Transmission and reflected loss versus input power of a single sweep for various durations.

These measurements were repeated for other sweep durations, and in all cases, regardless of the sweep duration, the response saves its shape at the same power. This confirms the above conclusion that switching time of the device is not longer than $75 \mathrm{~ns}$.

The results of the measurements carried out at the central frequency for transmission and return losses versus input power are presented in Fig. 11. From this figure, it can be seen that the behavior of the transmission losses versus input power does not depend on sweep duration. As regards reflections, the measurements are more sensitive to variations in measurement conditions; so, these curves do not coincide. In any case, it can be seen that the behavior of transmission and return losses is similar to that observed in Fig. 3. This similarity reflects that every level of input power corresponds to its own value of HTS element conductivity.

\section{PoWer Limiter In Non-Linear Regime}

It is well-known that superconductor thin films are nonlinear. Many research works are devoted to the investigation of their nonlinear effects [12]-[14] and the nonlinear devices that utilize this effect. So, one evidently expects the same behavior by the presented power limiter in this regime too.

For investigating this, the scheme presented in Fig. 6 was modified by inserting behind the power limiter a bandpass filter with a rejection band at the frequencies of PL's operating bandwidth, which gives $60 \mathrm{~dB}$ suppress in the signal of the first harmonic.

The measurements revealed that no nonlinear harmonics appear in the PL output spectra at the levels of input signal up to at least $30 \mathrm{dBm}$. Such unusual behavior is, to our opinion, due to the device is selective and its transmission level at a frequency of the 3-d harmonic is around $-30 \mathrm{~dB}$. Besides, as the device is of reflection type, the power that takes part in nonlinear process is much less in comparison to that in the absorptive ones under similar conditions.

Microstrip resonator has one useful property: the irregularities of its strip conductor can be used to control the frequencies of its higher resonant modes. Therefore, by designing such PLs, one can easily suppress nonlinear harmonics, if required. 


\section{CONCLUSIONS}

A novel design of power limiter, based on a microstrip resonator and a HTSC element, is presented. The device has a structure of a three-pole bandpass filter, where HTSC element is a part of the middle resonator providing a coupling between the outer resonators when HTSC is in low-loss state. When the input power reaches a critical value, the HTSC element switches to the highloss state, and the $Q$-factor of the middle resonator drops. Consequent to this, the coupling between outer (input and output) resonators breaks, and incident power reflects from the device input. So, as the device operation is of reflective mode, instead of being of absorptive mode, the structure presented is sufficiently robust. This was demonstrated by fabricating $8 \mathrm{GHz}$ power limiter, which remained operable up to $40 \mathrm{~W}$ of input power with no degradation of the HTSC thin film. At this input power, $38 \mathrm{~dB}$ limitation was achieved. The proposed structure is a frequency selective device, i.e. a bandpass filter with $8.5 \%$ fractional bandwidth. When the PL begins to limit, the threshold level is $-5 \mathrm{dBm}$, regardless of whether the device operates in continuous sweep or in pulse mode. The switching time estimated from the pulse measurement appears to be not more than 75 ns. No nonlinear behavior of the device was observed with input power up to $30 \mathrm{dBm}$. We connect this fact with the device's construction and operation principles.

Operation of a device in a limited pass-band may seem to be a disadvantage. But, as a rule, radio-electronic systems, whose input circuits require protection, operate in a limited pass-band, besides having input filters. With such PL, we not only protect the input circuit of the system from overloading, but also filter interfering signals with a filter having low thermal noise. Thus, this property is rather an advantage of the PL described here.

\section{REFERENCES}

[1] D. M. Pozar, and D. H. Schaubert. "Comparison of architectures for monolithic phased array antennas". Microwave Journal. 1986, 93-104.

[2] Shigekazu Hori, Masahiro Kuroda, Kenji Kanema and Susumu Okano. "High-power MIC diode limiters for Sand X-band radars". Microwave Symposium Digest, 1979 IEEE MTT-S International, April 1979, Orlando, USA

[3] F. Jahan, M. Gaevski, J. Deng, R. Gaska, M. Shur and G. Simin. "RF power limiter using capacitively-coupled contacts III-nitride varactor". Electronics Letters, Vol. 48, No. 23, 2012.

[4] C. Collado, A. Hueltes, E. Rocas, J. Mateu, J. C. Booth, J. M. O'Callaghan, and J. Verdú. "Absorptive Limiter for Frequency-Selective Circuits." IEEE Microwave and Wireless Components Letters, Vol. 24, No. 6, June 2014, pp. 415-417

[5] P. Phudpong and I. C. Hunter. "Frequency-Selective Limiters Using Nonlinear Bandstop Filters." IEEE Transactions on Microwave Theory and Techniques, Vol. 57, No. 1, January 2009, pp. 157-164

[6] A.P.M. Maas, J.P.B. Janssen, F.E. van Vliet. "Set of XBand distributed absorptive limiter GaAs MMICs". Proceedings of the 4th European Radar Conference, October 2007, Munich Germany

[7] J. Gauvin, O. Vendier, J.-L. Cazaux and P. Blondy. "Microwave power limiters based on RF-MEMS". MEMS 2013, Taipei, Taiwan, January 20 - 24, 2013

[8] Yu.A. Budzinskiy, S.V. Bykovskiy, V.E. Kotov, O.A Savrukhin. "On the increase of the frequency band of cyclotron protective device". CriMiCo'2005, Sevastopol, Ukraine, September 2005

[9] Yu.A. Budzinskiy, S.V. Bykovskiy, V.E. Kotov, O.A Savrukhin. "On the increase of the frequency band of cyclotron protective device". CriMiCo'2014, Sevastopol, Ukraine, September 2014

[10] A. T. Findikoglu, D. W. Reagor, K. O. Rasmussen, A. R. Bishop, N. Gronbech-Jensen, Q. X. Jia, Y. Fan, C. Kwon, and L. A. Ostrovsky. "Electrodynamic properties of coplanar waveguides made from high-temperature superconducting $\mathrm{YBa}_{2} \mathrm{Cu}_{3} \mathrm{O}_{7-\delta}$ electrodes on nonlinear dielectric $\mathrm{SrTiO}_{3}$ substrates". Journal of Applied Physics, Vol. 86, No. 3, 1999, pp. 1558-1568

[11] J. C. Booth, D. A. Rudman, and R. H. Ono. "A SelfAttenuating Superconducting Transmission Line for Use as a Microwave Power Limiter". IEEE Transactions on Applied Superconductivity, Vol. 13, No. 2, 2003, pp. 305310

[12] A. A. Kalenyuk. "Nonlinear microwave response of a superconducting $\mathrm{YBa}_{2} \mathrm{Cu}_{3} \mathrm{O}_{7} \delta$ microstrip transmission line with a constriction". Low Temperature Physics, Vol. 35, No. 2, 2009, pp. 105-111

[13] A.A. Kalenyuk, S.I. Futimsky, A.L. Kasatkin, A.I.Gubin, S.A.Vitusevich. "Nonlinear attenuation in YBCO coplanar transmission line in applied magnetic field". $M S M W^{\prime} 13$, Kharkov, Ukraine, June 23-28, 2013

[14] O. G. Vendik, I. B. Vendik, and T. B. Samoilova. "Nonlinearity of Superconducting Transmission Line and Microstrip Resonator". IEEE Transactions on Applied Superconductivity, Vol. 45, No. 2. 1997, pp. 173-178

[15] N. T. Cherpak, A. A. Lavrinovich, A. I. Gubin, and S. A. Vitusevich. "Direct-current-assisted microwave quenching of $\mathrm{YBa}_{2} \mathrm{Cu}_{3} \mathrm{O}_{7-\delta}$ coplanar waveguide to a highly dissipative state". Applied Physics Letters, Vol. 105, 2014, pp. 022601

[16] M. M. Gajdukov, S G Kolesov, L Kowalewicz, A B Kozyrev, O G Vendik. "Microwave Power Limiter Based on High-Tc Superconductive Film". Electronics Letters, Vol. 26, No. 16, 1990, pp. 1229-1231

[17] A. B. Kozyrev." Phenomena of Fast Switching of Superconducting Films and its Applications to Microwave Microelectronics" Soros. Obrazov. Zh. Vol. 8, No. 1, 2004, pp. 93-100.

[18] E. Rocas, , A. Padilla, J. Mateu, N. Orloff, J. M. O'Callaghan, C. Collado, and J. C. Booth. "Superconducting Multiplexer Filter Bank for a FrequencySelective Power Limiter". IEEE Transactions on Applied Superconductivity, Vol. 21, No. 3, 2011, pp. 542-546

[19] B. A. Belyaev, I. V. Govorun, A. A. Leksikov, and A. M. Serzhantov. "Receiver Protecting Device Based on Microstrip Structure with High-Temperature Superconductor Film”. Technical Physics Letters, 2012, Vol. 38, No. 3, pp. 211-214

[20] V.V. Tyurnev. "Coupling Coefficients of Resonators in Microwave Filter Theory". Progress In Electromagnetics Research B, 2010, Vol. 21, pp. 47-67

[21] B. A. Belyaev, N. V. Laletin, and A. A. Leksikov. "Coupling Coefficients of Irregular Microstrip Resonators and Frequency-Selective Properties of a Two-Section Irregular-Microstrip-Resonator Filter". Journal of Communications Technology and Electronics, 2002, Vol. 47, No. 2, pp. 14-23

[22] F. Gao, Z. Zhou, and M. Feng, M. Scharen, A. Cardona, and R. Forse. "Hysteresis effect in microwave power transmission of high temperature superconducting coplanar transmission lines". Applied Physics Letters, Vol. 67, 1995, pp. 2229

[23] B.A. Belyaev, AM. Serzhantov,V.V. Tyurnev, Ya.F. Bal'va, A. A. Leksikov. "Planar Bandpass Filter with 100dB Suppression up to Tenfold Passband Frequency". Progress In Electromagnetics Research C, 2014, Vol. 48, 3744. 